\title{
Towards Regional Elastography of Intracranial Aneurysms
}

\author{
Simone Balocco ${ }^{1,2}$, Oscar Camara ${ }^{1,2}$, and Alejandro F. Frangi ${ }^{1,2}$ \\ ${ }^{1}$ Center for Computational Imaging \& Simulation Technologies in Biomedicine, \\ Universitat Pompeu Fabra, Barcelona, Spain \\ ${ }^{2}$ Networking Center on Biomedical Research (CIBER-BBN), Barcelona, Spain
}

\begin{abstract}
Weak spots in the aneurysm could be identified estimating the regional stiffness of the wall. Our approach consists in defining a parametric biomechanical model of the vessel which, given the patient's vascular morphology and the blood in- and outflow obtained from non-invasive imaging as well as parameters describing the local elasticity of the wall, enables the computation of the theoretical deformed wall position. The distance between this latter and the one obtained from the aneurysm pulsation is iteratively minimized in order to estimate the optimal set of stiffness parameters. In order to reduce the number of variables to estimate, the aneurysm morphology is clustered into a limited number of regions with uniform stiffness. A random noise perturbation $(<5 \mathrm{~mm})$ is applied to the reference deformations and strains, showing that the robustness of the clustering decreases to $75 \%$ and errors of the stiffness estimates remain below $10 \%$ of the reference values.
\end{abstract}

\section{Introduction}

A cerebral aneurysm is a vascular disease in which the weakness of the cerebral artery wall causes a localized dilation of the blood vessel and occasionally its rupture thus leading to stroke. The assessment of the aneurysm elasticity may allow the identification of potential rupture sites such as blebs [1] before a noticeable modification of the aneurysm shape and the periodical survey of structural changes of the wall predict disease progression. Although non-invasive estimation of tissue elasticity in vivo in other vascular segments has been demonstrated [2], these techniques have limited applicability in intracranial segments due to limited imaging resolution [1] since aneurysmal wall motion would require high spatio-temporal accuracy in $3 \mathrm{D}$ to be detected.

The elastic properties of vessels can be deduced indirectly using an inverse problem formulation. In our application, the inverse problem framework will consist in estimating the mechanical parameters of a biomechanical model of the aneurysm based on non-invasive measurements of the dynamic pulsation and blood flow. The first theoretical framework addressed to the elasticity estimation of cerebral aneurysms has recently been proposed by Kroon and Holzapfel [3]. The authors present a numerical model characterized by a spherical shape discretized into a small number of mesh elements and by boundary conditions based on a uniform distribution of the pressure along the surface. The feasibility of their approach was shown on synthetic data. 
In this work, we present a more realistic approach for the estimation of regional aneurysmal stiffness where, for the first time, patient-specific morphology and inflow curves are used to solve for the model parameters. For doing so, patient-specific vascular morphology and inflow curves are introduced into a biomechanical model of the arterial segment. Furthermore, we propose to couple fluid-dynamic and structural equations so that the non-uniform pressure distribution of the blood is taken into account for generating realistic wall pulsation.

The difficulty of reliably measuring the aneurysm 3D wall motion in vivo has lead us to the use of finite element analysis to generate the reference measurements needed to evaluate the proposed framework, considering the morphology and flow variations obtained from the patient. We made the assumption that the membrane had a piecewise distribution of uniform stiffness since different regions present different degree of rupture fragility. Therefore, we divided the aneurysm surface in three regions, namely the vessel (very low rupture risk), the bleb (high rupture risk) and the dome (low rupture risk), as shown in Figure 1. These regions of different elastic parameters will be recovered at the model parameter optimization stage with a region clustering technique applied to the strain maps of the measurements, which considerably speeds up the parameter optimization stage. In order to evaluate the accuracy of these registration techniques needed for elasticity estimation purposes, the robustness of the proposed inverse problem framework is studied by applying random noise perturbations to the deformation field and strain maps of the reference measurements.

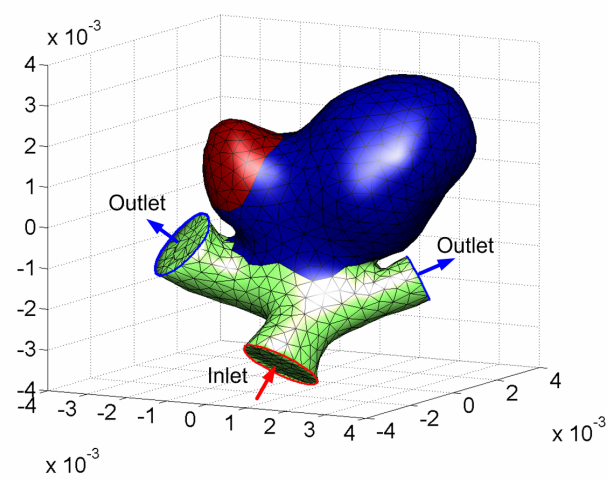

Fig. 1. Isometric view of the patient geometry. The surface is divided in three regions: the arterial vessel (green) the aneurysm dome (blue) and the bleb (red).

\section{Method}

Estimations of elasticity are obtained using an inverse problem formulation. A parametric biomechanical model of the vessel, given the definition of the initial shape obtained from angiographic imaging, boundary flow conditions based on the patient's blood inflow curve and a parameterization of the local elasticity of the wall, enables the computation of the theoretical deformed shape corresponding to such settings. 
The distance between this simulated deformed shape and the one obtained from wall motion measurements capturing the aneurysm pulsation is iteratively minimized in order to estimate the optimal set the biomechanical parameters (i.e. stiffness values). Finally, in order to reduce the number of parameters to estimate, the aneurysm shape is automatically clustered into a limited number of regions with uniform stiffness, corresponding to zones having different tissue characteristics.

\subsection{Biomechanical Model}

The biomechanical model generates a deformation field that will be applied to the initial aneurysm mesh, considered as the geometry at rest position, in order to obtain the surface of the aneurysm during the pulsation. This is achieved by coupling fluiddynamic equations describing the haemodynamics inside the vessel with structural equations describing the surface pulsation. The equations of the biomechanical model are solved by finite element analysis using the COMSOL Multiphysics $®$ v3.4 (COMSOL Inc., Burlington, MA, USA) ${ }^{1}$ software.

The relation between blood velocity and pressure for complex geometries and flow regimes is described by the Navier-Stokes non-linear equations:

$$
\rho \frac{\partial \vec{v}}{\partial t}-\nabla \eta\left(\nabla \overrightarrow{\boldsymbol{v}}+(\nabla \overrightarrow{\boldsymbol{v}})^{T}\right)+\rho(\nabla \overrightarrow{\boldsymbol{v}}) \overrightarrow{\boldsymbol{v}}+\nabla p=0
$$

where $\vec{v}$ is the time-dependent flow velocity at a point in $3 \mathrm{D}$ space, $p$ is the hydraulic pressure, $\rho$ is the mass density of blood and $\eta$ is the kinematics viscosity of the medium.

In order to estimate the blood pressure and the flow velocity inside the vessel, the gradients of the velocity $(\Delta \hat{v})$ and pressure $(\Delta \hat{p})$ between systole and diastole are defined as boundary conditions at the inlet and outlet of the vessel, respectively. Following the energy conservation principle, the hydrodynamic pressure calculated at each point of the surface is then dynamically applied to the aneurysm surface as a mechanical load along the cardiac cycle. The relationship between hydraulic pressure, wall tangential stress $(\sigma)$, local curvature ( $\kappa$ is the curvature radius) and thickness of the membrane wall $(h)$ is described by the Laplace's law: $\sigma=p / h \cdot \kappa$.

In this application, the membrane kinetics is modelled by the Hooke's law, which relates the stress and strain $(\varepsilon)$ tensors through the Young's modulus $(E): \sigma=E \cdot \varepsilon$.

Hence, the aneurysm wall is defined as linear elastic and isotropic material since we assume it undergoes small deformations, as proved by the fact they can hardly be detected using the existing imaging devices. The aneurysm wall is usually characterized by a thin membrane [3], thus we modelled it as a shell object in which the surface is composed by triangles [4] and the thickness is then a variable of the mesh element. As a consequence, the stiffness of a shell element $E^{*}$ will be obtained as the product between the Young's modulus and the thickness of the wall. Using such a surrogate of elasticity, which represents the composite stiffness of the three arterial tunicae, a membrane element with a low stiffness corresponds either to a region of

\footnotetext{
${ }^{1}$ http://www.comsol.com/
} 
small elasticity or small thickness. This ambiguity can be overcome by measuring the exact thickness at each point or assuming a uniform thickness around the surface.

\subsection{Inverse Problem Formulation}

Region clustering. The estimation of the stiffness for each element of the mesh implies that the number of variables to estimate is proportional to the number of mesh triangles making the numerical problem ill-conditioned when meshes with a large number of elements are used. The use of the strain maps obtained from measurements can partially solve this problem since a mode of the histogram of these strain maps corresponds to a region characterized by a specific tissue property. Therefore, we propose to recover those regions that are characterized by uniform stiffness with a region clustering technique applied to the strain maps in order to reduce the number of variables at the parameter optimization stage.

Strain is defined as the stretch of a surface element over its original length. For a given displacement field $\vec{d}$, the strain tensor is a symmetric matrix composed by the elements $\varepsilon_{i j}=1 / 2\left(\nabla_{i} d_{j}+\nabla_{j} d_{i}\right)$ where $\varepsilon_{i j}$ is the strain in the direction along the axis $i$ and $\nabla_{i} d_{j}$ is a differential of the component $d_{j}$ at any point in the direction $i$. The eigenvalues of $\mathcal{E}$, called principal strains $\left(\varepsilon_{1}, \varepsilon_{2}, \varepsilon_{3}\right)$, correspond to the strain in the direction of its maximum variation. Since the aneurysm surface is a quasi-spherical thin-walled object, one can assume that the first and the second principal strains approximate the maximum deformation in the tangential directions, whereas the third correspond to the maximum membrane thinning.

The region clustering will then consist in applying the k-means algorithm [5] to the three strain maps computed from measurements. The optimal number of clusters is automatically obtained with a technique based on cluster silhouettes [6]. The output of this step will be regions composed by the elements of the aneurysm surface mesh having similar strain values in each of the three strain maps. Elements at the border of two regions may be wrongly classified in a given strain map. Hence, the classification provided by the three strain maps is fused (most common value is chosen) to avoid these ambiguities.

Parameter estimation. In pulsatile flow conditions, the theoretical position of the aneurysm shape in systole $s_{s y s}^{\text {theo }}$ can be expressed at any point of the vessel wall as a function of the regional stiffness $E^{*}$ and of the corresponding blood flow according to the biomechanical model equations. Let $\phi$ be the error function, defined as the distance between the nodes of the theoretical surface generated by the biomechanical model and the closest point of the surface experimentally acquired in systole $s_{s y s}^{\text {exp }}$ :

$$
\phi=\sum_{n}\left\|s_{\text {sys }}^{\text {theo }}\left(E_{\text {est }}^{*}\right), s_{\text {sys }}^{\text {exp }}\right\|,
$$

where $\|$,$\| represents the Euclidean distance. Hence, the parameter estimation me-$ thod consists in minimizing $\phi$ using the Nelder-Mead simplex method [7] solving for the stiffness parameters of the biomechanical model. The initialization of the algorithm with reasonable values of $E^{*}$ from the literature is required to reduce the number of iterations needed to converge and the risk to get trapped by local minima of $\phi$. We considered the convergence of the optimization stage was reached when the gradient between two successive values of $\phi$ was lower than a given threshold. 


\section{Digital Phantom with Patient-Specific Boundary Conditions}

The reference measurements needed to evaluate the proposed inverse problem framework are generated through a numerical simulation (forward model) in which three regions of the aneurysm surface (vessel, dome and bleb) and their corresponding stiffness values $\left(E_{1}^{*}, E_{2}^{*}, E_{3}^{*}\right)$ were set according to literature. As described in Section 2, the inverse problem stage then recovers the number and the location of these regions, using the patient-specific measurements provided by the forward problem and iteratively estimating the parameters of the biomechanical model. The robustness of the method in the presence of imprecise measurements is evaluated by adding noise to the deformation fields and strain maps issued from the forward problem simulation. Schweiger et al. [8] proposed a similar evaluation methodology for their inverse problem approach to the estimation of volume change in brain images.

\subsection{Forward Model}

The aneurysm mesh, composed by 5136 triangle elements, is obtained segmenting the aneurysm in a patient CTA image using a level-set based method [9]. A volumetric mesh is needed for the fluid-dynamic computation, and it is automatically generated from the surface triangulation using COMSOL, resulting in a 3D mesh with 19,299 tetrahedral elements. Finally, three regions of the surface mesh, representing the arterial vessel, the dome and the bleb of the aneurysm (Figure 1), were defined.

Literature values of thickness and elasticity of the tissues (see Table 1) assessed by histological sampling of the communicating artery (PCoA) [10] have been used for characterizing the mechanical properties of the aneurysm wall. To the best of our knowledge, no in vivo measurements of the ratio between the elasticity and wall thickness of vessel dome and bleb before aneurysm rupture are available in the literature. However, MacDonald [11] showed, in an ex vivo study, that the thickness of different regions of certain aneurysms presented a ratio of around 3 . Hence, we assume that the material constituting the vessel is stretched for developing the aneurysm, leading to a reduction (around one fourth) of the thickness ratio between the vessel area and the aneurysm wall. Assuming the elasticity properties of the tissue do not change, this thinning implies a corresponding reduction of the aneurysm wall stiffness. Furthermore, we assumed that the bleb had a weaker wall, thus its Young's modulus was set to one sixth of the dome, as shown in Table 1.

Table 1. Mechanical reference parameters $\left(E_{r e f} *\right)$ used in the forward model stage

\begin{tabular}{|c|c|c|c|c|c|}
\hline & $\begin{array}{c}\text { Label } \\
E_{\text {ref }}{ }^{*}\end{array}$ & $\begin{array}{c}\text { Poisson } \\
\text { ratio }\end{array}$ & $\begin{array}{c}\text { Young's } \\
\text { modulus } E\end{array}$ & $\begin{array}{c}\text { Thickness } \\
h\end{array}$ & $\begin{array}{c}\text { Stiffness } \\
E^{*}\end{array}$ \\
\hline Vessel & $E_{1}{ }^{*}$ & 0.45 & $1.6 \mathrm{MPa}$ & $0.018 \mathrm{~cm}$ & $288[\mathrm{~Pa} \mathrm{~m}]$ \\
\hline Dome & $E_{2}{ }^{*}$ & 0.45 & $1.6 \mathrm{MPa}$ & $0.0045 \mathrm{~cm}$ & $72[\mathrm{~Pa} \mathrm{~m}]$ \\
\hline Bleb & $E_{3}{ }^{*}$ & 0.45 & $0.27 \mathrm{MPa}$ & $0.0045 \mathrm{~cm}$ & $28.8[\mathrm{~Pa} \mathrm{~m}]$ \\
\hline
\end{tabular}

The boundary conditions $\Delta \hat{v}$ and $\Delta \hat{p}$ are estimated by measuring the mean velocity variation using a MRA Q-flow technique from a 1.5T Intera MRI scanner (Philips Medical Systems, Best, The Netherlands) and the blood pressure with a brachial sphygmomanometer on the patient, respectively. The gradient between the minimum 
and the maximum values of the velocity waveform (from 0.18 to $0.38 \mathrm{~m} / \mathrm{s}$ ) and the corresponding pressure measurements (from 97 to $133 \mathrm{mmHg}$ ) were employed as boundary conditions.

The simulated deformed shape $s_{s y s}^{\text {theo }}$ and the pressure distribution at the systolic peak were computed by solving the biomechanical equation described in Section 2 using the COMSOL's finite element software.

Figure 2-a illustrates the relative blood pressure between inlet and outlet of the aneurysm at the systolic peak. This figure shows that the pressure distribution is nonuniform and highly influenced by the flow stream patterns and by the impingement jets induced by the patient-specific geometry. The magnitude of the displacements is mapped into the original shape in Figure $2 b$.

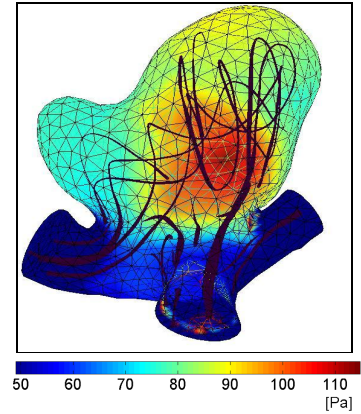

(a)

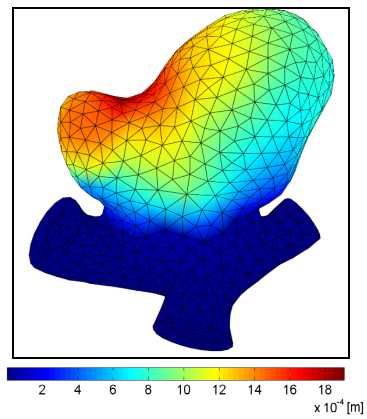

(b)

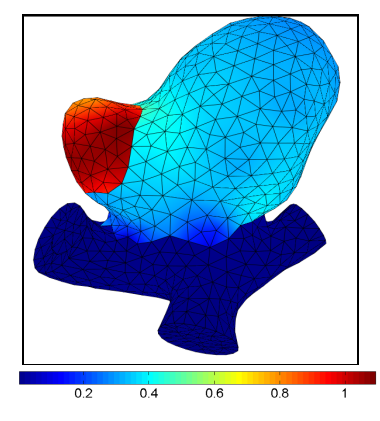

(c)

Fig. 2. Relative pressure distribution (a) superimposed to the flow streamlines at the systolic peak. Displacement magnitude (b) and principal strain $\mathcal{E}_{1}$ (c) distributions at the systolic peak.

\subsection{Parameter Estimation}

In this stage, the three principal strain distributions (Figure 2c) are computed from the displacement field generated by the forward model. Sharp gradients correlating with the different region contours appear in the strain maps, which are not visible in the displacement distributions. This is due to the fact that strain measures tissue stretching, which is abrupt where the two tissues connect. The identification of the three different regions with uniform elasticity is consequently achieved applying the region clustering technique described in Section 2.2 to the strain maps.

At the optimization stage, convergence is reached after 60 iterations using the mean value of $E_{1}^{*}, E_{2}^{*}$ and $E_{3}^{*}$ as initialization of the stiffness of the three regions. The whole process takes around 15 minutes on a dual-core Pentium IV PC.

The robustness of the region clustering and the parameter optimization was evaluated separately; applying normally distributed random noise of amplitude $w$ to the displacement and strain fields generated by the forward simulation.

The clustering technique identify the three regions, and all mesh elements are correctly assigned to the correct region in the absence of noise, as can be seen in Figure 3 -a. When the noise amplitude applied to the strain increases, thus blurring region borders, the robustness of the clustering technique decreases to $85 \%$ for the dome and 


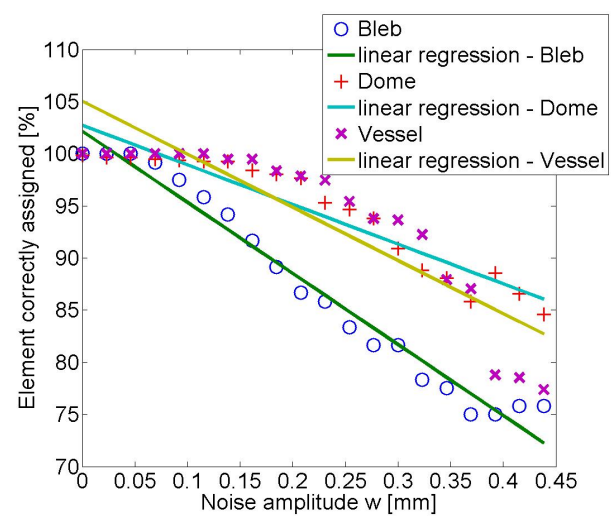

(a)

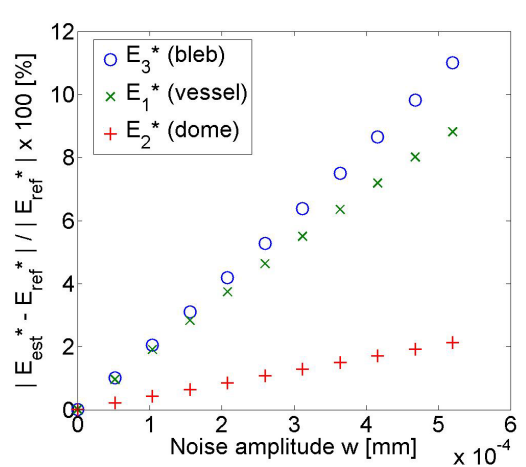

(b)

Fig. 3. Percentage of mesh elements correctly assigned to a region (a) and stiffness errors (b) as a function of noise

vessel regions and to $75 \%$ for the bleb area. Indeed, the clustering errors primarily affect the bleb, and have more influence on the mesh elements lying close to the interface. The error in the estimation of the stiffness in each region of the aneurysm increases linearly with the additive noise applied to the displacement field generated by forward problem simulation (Figure 3-b) and up to $5 \mathrm{~mm}$ of noise is lower than $10 \%$. The higher slope corresponds to the estimation of the bleb stiffness since it is strongly affected by the noise applied to the dome, and the vessel itself where the lower simulated displacements are present.

\section{Conclusion and Discussion}

In this paper a method for the assessment of the elastic properties of cerebral aneurysms using an inverse problem framework and patient-specific boundary conditions is presented. The evaluation of the method accuracy and robustness was performed simulating the reference wall motion and perturbing the measurements with additive noise. Measurement errors primarily affect the regions with lower stiffness since they are characterized by the largest displacements. The estimation of the bleb stiffness becomes unreliable with noise amplitudes larger than two.

Potential clinical applications of this technique such as the identification of weak spots in the vessel wall related to rupture events and the early identification of bleb development may be possible due to the low computational cost of the proposed technique.

Future work will focus on an in vitro validation of the method performed on phantoms. In terms of the model, we will study the incorporation of the anisotropy of the membrane [3] and the use of a different model for forward and inverse problems.

\section{Acknowledgments}

This work was partially supported in the context of the CDTI CENIT-CDTEAM and EC @neurIST (IST-FP6-2004-027703) projects. The work of OC is supported by the 
Spanish MICINN under a Ramon y Cajal Research Fellowship. The authors would like to acknowledge G. Blasco and S. Pedraza, MD, PhD, from the Hospital Josep Trueta (Girona, Spain) for providing the MRA flow measurements.

\section{References}

1. Hayakawa, M., Katada, K., Anno, H., Imizu, S., Hayashi, J., Irie, K., Negoro, M., Kato, Y., Kanno, T., Sano, H.: CT Angiography with Electrocardiographically Gated Reconstruction for Visualizing Pulsation of Intracranial Aneurysms: Identification of Aneurysmal Protuberance Presumably Associated with Wall Thinning. Am. J. Neuroradiol. 26, 1366-1369 (2005)

2. Zhang, X., Kinnick, R., Fatemi, M., Greenleaf, J.: Noninvasive Method for Estimation of Complex Elastic Modulus of Arterial Vessels. IEEE Trans. Ultrason. Ferroel. Freq. Contr. 52, 642-652 (2005)

3. Kroon, M., Holzapfel, G.A.: Estimation Of The Distributions Of Anisotropic, Elastic Properties And Wall Stresses Of Saccular Cerebral Aneurysms By Inverse Analysis. Proc. R. Soc. Lond. A. (in press)

4. Argyris, J.H., Fried, I., Scharpf, D.W.: The TUBA family of plate elements for the matrix displacement method. J. Roy. Aeronaut. 72, 701-709 (1968)

5. Hartigan, J.A.: Clustering Algorithms. John Wiley \& Sons, New York (1975)

6. Kaufman, L., Rousseeuw, P.J.: Finding Groups in Data: An Introduction to Cluster Analysis, New York (1990)

7. Nelder, J.A., Mead, R.: A Simplex Method for Function Minimization. Computer Journal 7, 308-313 (1965)

8. Schweiger, M., Camara-Rey, O., Crum, W.R., Lewis, E., Schnabel, J.A., Arridge, S.R., Hill, D.L.G., Fox, N.C.: An Inverse Problem Approach to the Estimation of Volume Change. In: Duncan, J.S., Gerig, G. (eds.) MICCAI 2005. LNCS, vol. 3750, pp. 616-623. Springer, Heidelberg (2005)

9. Hernandez, M., Frangi, A.F.: Non-parametric Geodesic Active Regions: Method and evaluation for cerebral aneurysms segmentation in 3DRA and CTA. Med. Image Anal. 11, 224-241 (2007)

10. Alastruey, J., Parker, K., Peiró, J., Byrd, S., Sherwin, S.: Modelling the circle of Willis to assess the effects of anatomical variations and occlusions on cerebral flows. J. Biomech. 40, 1794-1805 (2007)

11. MacDonald, D.J., Finlay, H.M., Canham, P.B.: Directional Wall Strength in Saccular Brain Aneurysms from Polarized Light Microscopy. Annals of Biomedical Engineering 28, 533-542 (2000) 